\title{
A CHARACTERISTIC PROPERTY OF QUADRATIC RESIDUES
}

\author{
JOHN B. KELLY
}

1. Introduction. Let $p$ be an odd prime. We denote by $R_{p}$ the set of quadratic residues $(\bmod p)$, by $N_{p}$ the set of quadratic nonresidues, and by $r_{i}, i=1,2, \cdots,(p-1) / 2$, and $n_{j}, j=1,2, \cdots,(p-1) / 2$, the elements of $R_{p}$ and $N_{p}$, respectively. We shall indicate by $r+N_{p}$ the set of all residues $(\bmod p)$ obtained by adding the (fixed) quadratic residue, $r$, to the various elements of $N_{p}$. A similar significance attaches to such expressions as $r+R_{p}, n+R_{p}$, and $n+N_{p}$.

The following two theorems are well known.

THEOREM 1. Let $p$ be of the form $4 k+1, r$ an arbitrary quadratic residue, $n$ an arbitrary nonresidue. The sets $r+N_{p}$ and $n+R_{p}$ consist of $k$ quadratic residues and $k$ quadratic nonresidues.

THEOREM 2. Let $p$ be of the form $4 k-1, r$ an arbitrary quadratic residue, $n$ an arbitrary nonresidue. The sets $r+N_{p}$ and $n+R_{p}$ consist of $0, k-1$ quadratic residues, and $k-1$ nonresidues.

One may ask whether or not the "equidistribution" property mentioned in Theorems 1 and 2 actually characterizes the quadratic residues among subsets of $(p-1) / 2$ nonzero elements of the cyclic group of order $p$. It is also natural to inquire whether or not there exist subsets with this property when we replace the prime modulus, $p$, by a composite modulus, $n$. These questions are answered by the two theorems which follow.

THEOREM 3. Let $m$ be an integer of the form $4 k+1$. Let the least positive residues mod $m$ be divided into two mutually exclusive classes of $2 k$ elements each. Call these classes $A$ and B. Suppose that $A$ and B may be chosen so that:

(a) $1 \in A$.

(b) For every choice of $a^{*} \in A$, the set $a^{*}+B$ contains $k$ elements of $A$ and $k$ elements of $B$.

(c) For every choice of $b^{*} \in B$, the set $b^{*}+A$ contains $k$ elements of $A$ and $k$ elements of $B$.

Then:

(1) $m$ is a prime.

(2) $A$ consists of the quadratic residues $\bmod m$ and $B$ consists of the quadratic nonresidues mod $m$.

Presented to the Society, December 29, 1953; received by the editors March 7, 1953. 
THEOREM 4. Let $m$ be an integer of the form $4 k-1$. Let the $4 k-2$ least positive residues $\bmod m$ be divided into two mutually exclusive classes of $2 k-1$ elements each. Call these classes $A$ and $B$. Suppose that $A$ and $B$ may be chosen so that:

(a') $1 \in A$.

( $\left.\mathrm{b}^{\prime}\right)$ For every choice of $a^{*} \in A$, the set $a^{*}+B$ contains $0, k-1$ elements of $A$, and $k-1$ elements of $B$.

Then:

(1') $m$ is a prime.

$\left(2^{\prime}\right) A$ consists of the quadratic residues mod $m$ and $B$ consists of the quadratic nonresidues $\bmod m$.

Hypothesis (b) of Theorem 3 implies:

(d) If $a \in A$, then $m-a \in A$;

while hypothesis $\left(\mathrm{b}^{\prime}\right)$ of Theorem 4 implies:

( $\left.\mathrm{d}^{\prime}\right)$ If $a \in A$, then $m-a \in B$; in other words, $B=-A$.

The analogue, $\left(\mathrm{c}^{\prime}\right)$, of $(\mathrm{c})$, is an immediate consequence of $\left(\mathrm{b}^{\prime}\right)$ and $\left(d^{\prime}\right)$.

It is of some interest to observe that the hypotheses of Theorems 3 and 4 involve only the additive group $(\bmod m)$ whereas the conclusion involves the multiplicative group. This is not overly surprising, perhaps, when one recalls that the multiplicative group $(\bmod m)$ is isomorphic to the group of automorphisms of the additive group $(\bmod m)$.

The main part of this paper, $\S 3$, is concerned with the proof of Theorem 3. The proof of Theorem 4 so closely parallels the proof of Theorem 3 that we have not included it. For the sake of completeness, we have given a proof of Theorem 1 in $\$ 2$, inasmuch as neither this theorem nor Theorem 2 is explicitly stated in readily available sources. Again, since the proof of Theorem 2 so closely resembles that of Theorem 1, we have seen fit to omit it.

We conclude with some remarks $(\$ 4)$ on the extension of our results to finite fields, and on some work of Perron closely related to ours.

2. Proof of Theorem 1. Let $p=4 k+1$. Consider the set, $H_{p}$, of all expressions of the form $r_{i}+n_{j}, i=1,2, \cdots,(p-1) / 2, j=1,2, \cdots$, $(p-1) / 2$. We show that all nonzero residues are represented equally often in $H_{p}$. ( 0 is not represented, since $r \in R_{p}$ implies $p-r \in R_{p}$ when $p \equiv 1(\bmod 4)$.) To every representation of $1,1=r+n$, corresponds a unique representation of $g, g=r^{\prime}+n^{\prime}$, where $r^{\prime}=g r$ and $n^{\prime}=g n$ when $g$ is a quadratic residue and $r^{\prime}=g n, n^{\prime}=g r$ when $g$ is a nonresidue. Conversely, to every representation of $g, g=r^{\prime}+n^{\prime}$, cor- 
responds a unique representation of $1,1=r+n$, where $r=g^{-1} r^{\prime}$, $n=g^{-1} n^{\prime}$ when $g$ is a quadratic residue and $r=g^{-1} n^{\prime}, n=g^{-1} r^{\prime}$ when $g$ is a nonresidue. Thus a one-to-one correspondence exists between the representations of 1 and the representations of any other nonzero residue. Hence $H_{p}$ contains as many representations of quadratic residues as of nonresidues.

Suppose now that the set $1+N_{p}$ contained more (fewer) quadratic residues than nonresidues. Then the set $r_{i}+N_{p}=r_{i}\left(1+r_{i}^{-1} N_{p}\right)$ $=r_{i}\left(1+N_{p}\right)$ would also contain more (fewer) quadratic residues than nonresidues. Consequently $H_{p}=U_{i}\left(r_{i}+N_{p}\right)$ would contain more (fewer) quadratic residues than nonresidues, a contradiction.

It follows that the set $1+N_{p}$ contains as many quadratic residues as nonresidues; the sets $r_{i}+N_{p}=r_{i}\left(1+N_{p}\right)$ and $n_{j}+R_{p}=n_{j}\left(1+N_{p}\right)$ also have this property.

3. Proof of Theorem 3. Theorem 3 is considerably more difficult to prove than Theorem 1, even though it may be regarded as a converse of Theorem 1. We shall discuss the reason for this situation in $\$ 4$. Our principal tool is cyclotomy.

We define the symbol

$$
\left\{\begin{array}{c}
l, A \\
m
\end{array}\right\}
$$

as follows:

$$
\begin{aligned}
& \left\{\begin{array}{c}
l, A \\
m
\end{array}\right\}=1, \quad l \equiv l^{\prime}(\bmod m), \quad l^{\prime} \in A ; \\
& \left\{\begin{array}{c}
l, A \\
m
\end{array}\right\}=-1, \quad l \equiv l^{\prime}(\bmod m), \quad l^{\prime} \in B ; \\
& \left\{\begin{array}{c}
l, A \\
m
\end{array}\right\}=0, \quad l \equiv 0(\bmod m) .
\end{aligned}
$$

It follows from (b), (c), and (d) that

$$
\begin{array}{ll}
\sum_{j \in A}\left\{\begin{array}{c}
n-j, A \\
m
\end{array}\right\}=\frac{-1-\left\{\begin{array}{c}
n, A \\
m
\end{array}\right\}}{2}, & n \neq 0(\bmod m) ; \\
\sum_{j \in A}\left\{\begin{array}{c}
n-j, A \\
m
\end{array}\right\}=\frac{m-1}{2}, & n \equiv 0(\bmod m) .
\end{array}
$$


Let $\omega$ be any algebraic integer with the properties

$$
\omega^{m}=1 ; \quad \omega \neq 1 \text {. }
$$

Let

$$
\alpha(\omega, A)=\sum_{n=0}^{m-1}\left\{\begin{array}{c}
n, A \\
m
\end{array}\right\} \omega^{n}
$$

and

$$
\beta(\omega, A)=\sum_{j \in A} \omega^{i} .
$$

Then, using (e), (f), and ( $\mathrm{g}$ ), we have

$$
\begin{aligned}
\alpha(\omega, A) \beta(\omega, A) & =\sum_{j \in A} \sum_{n=0}^{m-1}\left\{\begin{array}{c}
n, A \\
m
\end{array}\right\} \omega^{n+i}=\sum_{n=1}^{m} \omega^{n} \sum_{j \in A}\left\{\begin{array}{c}
n-j, A \\
m
\end{array}\right\} \\
& =\frac{m-1}{2}+\sum_{n=1}^{m-1} \frac{-1-\left\{\begin{array}{c}
n, A \\
m
\end{array}\right\}}{2} \omega^{n} \\
& =\frac{-\alpha(\omega, A)}{2}+\frac{m}{2} .
\end{aligned}
$$

Hence $\alpha(\omega, A)(2 \beta(\omega, A)+1)=m$. But $2 \beta(\omega, A)+1=2 \beta(\omega, A)+1$ $-\sum_{j=0}^{m-1} \omega^{j}=\alpha(\omega, A)$. Thus $\alpha^{2}(\omega, A)=m$ and

$$
\alpha(\omega, A)= \pm m^{1 / 2} \text {. }
$$

Since the quadratic residues of a prime $p \equiv 1(\bmod 4)$ have, according to Theorem 1, the properties (a), (b), (c), and (d), it follows that

$$
\sum_{n=0}^{p-1}\left[\frac{n}{p}\right] \omega^{n}=n \pm " p^{1 / 2}
$$

where

$$
\left[\frac{n}{p}\right]
$$

is the Legendre symbol and $\omega$ is a primitive $p$ th root of unity. Actually the indeterminacy of sign may be eliminated, but this requires a deeper analysis than is necessary for the proof of our theorem.

It is obvious that

$$
\alpha(\omega, A)=-\alpha(\omega, B) .
$$


We prove now that $m$ is a prime. If $m$ is neither a square nor a power of a prime, we obtain a contradiction fairly readily. For in this case we may put $m=p^{\lambda} Q$ where $p$ is a prime, $(p, Q)=1$, and $Q$ is not a square. If we let $\omega=e^{2 \pi i / p}$, a comparison of $(\mathrm{h})$ and $(\mathrm{j})$ reveals that the field $R(\omega)$ contains quadratic irrationalities other than $( \pm p)^{1 / 2}$, which is impossible.

Case 1. $m$ is a square. We may put $m=p^{2 t} Q^{2}$, where $p$ is a prime and $(p, Q)=1$. Let $\omega=e^{2 \pi i / p}$ and

$$
r_{j}^{(1)}=\sum_{i \equiv j(\bmod p)}\left\{\begin{array}{c}
i, A \\
m
\end{array}\right\} .
$$

Then

$$
\alpha(\omega, A)=\sum_{j=0}^{p-1} r_{j}^{(1)} \omega^{j}
$$

Since $1=-\sum_{j=1}^{p-1} \omega^{j}$, we have

$$
\alpha(\omega, A)=\sum_{j=1}^{p-1}\left(r_{j}^{(1)}-r_{0}^{(1)}\right) \omega^{j} .
$$

It follows from $(j)$ that

$$
\sum_{j=1}^{p-1}\left(r_{j}^{(1)}-r_{0}^{(1)}\right) \omega^{j}= \pm p^{t} Q= \pm p^{t} Q \sum_{j=1}^{p-1} \omega^{j} .
$$

The irreducibility of the cyclotomic polynomial entails the linear independence of $\omega, \omega^{2}, \omega^{3}, \cdots, \omega^{p-1}$, over the rational field.

Hence either

$$
r_{j}^{(1)}-r_{0}^{(1)}=p^{t} Q, \quad j=1,2,3, \cdots, p-1,
$$

or

$$
r_{j}^{(1)}-r_{0}^{(1)}=-p^{t} Q, j=1,2,3, \cdots, p-1 .
$$

Now

$$
\sum_{j=0}^{p-1} r_{j}^{(1)}=\sum_{n=1}^{m}\left\{\begin{array}{c}
n, A \\
m
\end{array}\right\}=0 .
$$

Thus $p r_{0}^{(1)} \pm p^{t} Q(p-1)=0$, whence $r_{0}^{(1)}= \pm p^{t-1} Q(p-1)$, and $r_{j}^{(1)}$ $= \pm p^{t-1} Q, j=1,2, \cdots, p-1$. In particular

$$
r_{1}^{(1)}= \pm p^{t-1} Q \text {. }
$$

Now let $\omega=e^{2 \pi i / p^{2}}$ and 


$$
r_{j}^{(2)}=\sum_{i \equiv j\left(\bmod p^{2}\right)}\left\{\begin{array}{c}
i, A \\
m
\end{array}\right\}, \quad j=0,1,2, \cdots, p^{2}-1
$$

Then

$$
\alpha(\omega, A)=\sum_{n=0}^{m-1}\left\{\begin{array}{c}
n, A \\
m
\end{array}\right\} \omega^{n}=\sum_{j=0}^{p^{2}-1} r_{j}^{(2)} \omega^{j}
$$

Using

$$
\begin{gathered}
1=-\omega^{p}-\omega^{2 p}-\cdots-\omega^{p^{2}-p}, \\
\omega=-\omega^{p+1}-\omega^{2 p+1}-\cdots-\omega^{p^{2}-p+1}, \\
. . . . . . . . . . . . \\
\omega^{p-1}=-\omega^{2 p-1}-\omega^{3 p-1}-\cdots-\omega^{p^{2}-1},
\end{gathered}
$$

and observing that each power of $\omega$ from $\omega^{p}$ to $\omega^{p^{2}-1}$ occurs just once on the right-hand side in this scheme, we find that

$$
\alpha(\omega, A)=\sum_{j=p}^{p^{2}-1}\left(r_{j}^{(2)}-r_{k(j)}^{(2)}\right) \omega^{j}
$$

where $k(j) \equiv j(\bmod p), k(j)=0,1,2, \cdots, p-1$. It follows from $(\mathrm{j})$ that $\sum_{j=p}^{p^{2}-1}\left(r_{j}^{(2)}-r_{k(j)}^{(2)}\right) \omega^{j}= \pm p^{t} Q= \pm p^{t} Q \sum_{l=1}^{p-1} \omega^{l p}$. The irreducibility of the cyclotomic polynomial of order $p^{2}$ entails the linear independence of $\omega^{p}, \omega^{p+1}, \omega^{p+2}, \cdots, \omega^{p^{2}-1}$ over the rational field. Hence

$$
r_{j}^{(2)}-r_{k(j)}^{(2)}=0, \quad \text { if } j \neq 0(\bmod p) .
$$

Thus

$$
r_{1}^{(2)}=r_{p+1}^{(2)}=r_{2 p+1}^{(2)}=\cdots=r_{p^{2}-p+1}^{(2)} .
$$

Now $r_{1}^{(1)}=\sum_{l=0}^{p-1} r_{l p+1}^{(2)}=p r_{1}^{(2)}$. From (m) we obtain

$$
r_{1}^{(2)}= \pm p^{t-2} Q \text {. }
$$

We continue this procedure, defining $r_{j}^{(3)}, r_{j}^{(4)}, \cdots, r_{j}^{(h)}, \cdots$ in an obvious way, putting successively $\omega=e^{2 \pi i / p^{3}}, \omega=e^{2 \pi i / p^{4}}, \cdots, \omega$ $=e^{2 \pi i / p^{h}}, \cdots$ in $(\mathrm{h})$, and using the irreducibility of the cyclotomic polynomials of orders $p^{3}, p^{4}, \cdots, p^{h}, \cdots$ to obtain the formula

$$
r_{1}^{(h)}= \pm p^{t-h} Q
$$$$
h \leqq 2 t \text {. }
$$

It is permissible to take $h=t+1$, for $t+1 \leqq 2 t$, so that $\omega=e^{2 \pi i / p^{t+1}}$ is an $m$ th root of unity. But then (o) implies that $r_{1}^{(t+1)}$ is not an integer, a contradiction. 
Case 2. $m$ is a power of a prime. Even powers of primes have been covered in Case 1 , so that we may suppose $m=p^{2 t+1}, p \equiv 1(\bmod 4)$, $t \geqq 1$. Let $\omega=e^{2 \pi i / p}$ and

$$
r_{j}^{(1)}=\sum_{i \equiv j(\bmod p)}\left\{\begin{array}{c}
i, A \\
m
\end{array}\right\} .
$$

Then, just as in Case 1 we have

$$
\alpha(\omega, A)=\sum_{n=0}^{m-1}\left\{\begin{array}{c}
n, A \\
m
\end{array}\right\} \omega^{n}=\sum_{j=0}^{p-1} r_{j}^{(1)} \omega^{j}=\sum_{j=1}^{p-1}\left(r_{j}^{(1)}-r_{0}^{(1)}\right) \omega^{j},
$$

whence, from $(j)$,

$$
\sum_{j=1}^{p-1}\left(r_{j}^{(1)}-r_{0}^{(1)}\right) \omega^{j}= \pm p^{t} p^{1 / 2} .
$$

But from (k) there follows

$$
p^{i} \sum_{j=1}^{p-1}\left[\frac{j}{p}\right] \omega^{j}= \pm p^{t} p^{1 / 2}
$$

so that

$$
\sum_{j=1}^{p-1}\left(r_{j}^{(1)}-r_{0}^{(1)}\right) \omega^{j}= \pm \sum_{j=1}^{p-1} p^{t}\left[\frac{j}{p}\right] \omega^{j} .
$$

The linear independence of $\omega, \omega^{2}, \cdots, \omega^{p-1}$ over the rational field implies that either

$$
r_{j}^{(1)}-r_{0}^{(1)}=p^{i}\left[\frac{j}{p}\right], \quad j=1,2, \cdots, p-1,
$$

or

$$
r_{j}^{(1)}-r_{0}^{(1)}=-p^{t}\left[\frac{j}{p}\right], \quad j=1,2, \cdots, p-1 .
$$

In either case,

$$
\sum_{j=0}^{p-1} r_{j}^{(1)}=p r_{0}^{(1)} \pm p^{t} \sum_{j=1}^{p-1}\left[\frac{j}{p}\right]=p r_{0}^{(1)}
$$

As before, $\sum_{j=0}^{p-1} r_{j}^{(1)}=0$. Hence $r_{0}^{(1)}=0$ and

$$
r_{1}^{(1)}= \pm p^{t} \text {. }
$$

Now let $\omega=e^{2 \pi i / p^{2}}$. Let 


$$
r_{j}^{(2)}=\sum_{j \geqq i\left(\bmod p^{2}\right), 0 \leqq i<m}\left\{\begin{array}{c}
i, A \\
m
\end{array}\right\} .
$$

Then, as before,

$$
\sum_{j=0}^{p^{2}-1} r_{j}^{(2)} \omega^{j}=\sum_{j=p}^{p^{2}-1}\left(r_{j}^{(2)}-r_{k(j)}^{(2)}\right) \omega^{j}= \pm p^{t} p^{1 / 2},
$$

where $k(j) \equiv j(\bmod p)$ and $k(j)=0,1,2, \cdots, p-1$. But from $(\mathrm{k})$ there follows

$$
p^{t} \sum_{l=1}^{p-1}\left[\frac{l}{p}\right] \omega^{l p}= \pm p^{t} p^{1 / 2}
$$

whence

$$
\sum_{j=p}^{p^{2}-1}\left(r_{j}^{(2)}-r_{k(j)}^{(2)}\right) \omega^{j}= \pm \sum_{l=1}^{p-1} p^{t}\left[\frac{l}{p}\right] \omega^{l p} .
$$

The linear independence of $\omega^{p}, \omega^{p+1}, \cdots, \omega^{p^{2}-1}$ over the rational field implies that

$$
r_{j}^{(2)}-r_{k(j)}^{(2)}=0, \quad \text { if } j \neq \equiv(\bmod p) .
$$

Hence

$$
r_{1}^{(2)}=r_{p+1}^{(2)}=r_{2 p+1}^{(2)}=\cdots=r_{p^{2}-p+1}^{(2)} .
$$

Now $r_{1}^{(1)}=\sum_{l=0}^{p-1} r_{l p+1}^{(2)}=p r_{1}^{(2)}$. From (p) we obtain

$$
r_{1}^{(2)}= \pm p^{t-1} \text {. }
$$

Just as with Case 1 we may continue this procedure to obtain the formula

$$
r_{1}^{(h)}= \pm p^{t-h+1}, \quad h \leqq 2 t+1 .
$$

In (r) it is permissible to take $h=t+2$, since $t+2 \leqq 2 t+1$ if $t \geqq 1$. Again, (r) implies that $r_{1}^{(h)}$ is not an integer, a contradiction.

We have thus shown that $m$ is a prime. We shall denote this prime by $p$. The proof of (2) is almost immediate. Suppose there were two distinct splittings of the nonzero residues $\bmod p$ with the properties described in the statement of the theorem. Call the corresponding pairs of sets $A, B$ and $A^{\prime}, B^{\prime}$. It follows from (j) that either $\alpha(\omega, A)=\alpha\left(\omega, A^{\prime}\right)$ or $\alpha(\omega, A)=\alpha\left(\omega, B^{\prime}\right)$. But, looking at (h) we see that either of these equations would contradict the linear independence of $\omega, \omega^{2}, \omega^{3}, \cdots, \omega^{p-1}$ over the rational field. 
4. Finite fields. Our proof of Theorem 1 used nothing more than the fact that the residues $\bmod p$ form a finite field. Hence Theorems 1 and 2 have obvious analogues for the Galois fields $G F\left(p^{n}\right)$. The analogue of Theorem 1 will hold if $p^{n} \equiv 1(\bmod 4)$; otherwise the analogue of Theorem 2 will hold.

We show, by means of a counter-example, that the expected analogues of Theorems 3 and 4 do not hold for finite fields in general. That is, there exist splittings of the nonzero elements of $G F\left(p^{n}\right)$ other than the splitting into squares and nonsquares which have the equidistribution property.

Consider the finite field, $G F\left(3^{2}\right)$, generated over the field of residues $(\bmod 3)$ by a solution, $\lambda$, of the irreducible equation $\lambda^{2}+1=0$. The square elements of $G F\left(3^{2}\right)$ are then $1,2, \lambda$, and $2 \lambda$; the nonsquares are $1+\lambda, 1+2 \lambda, 2+\lambda$ and $2+2 \lambda$. One can readily verify, however, that the splitting $(1,2,1+\lambda, 2+2 \lambda),(\lambda, 2 \lambda, 1+2 \lambda, 2+\lambda)$ also has the equidistribution property.

These considerations indicate that in proving parts 2 and $2^{\prime}$ of Theorems 3 and 4, we must use more than the fact that the residues $(\bmod p)$ form a finite field. Our use of cyclotomy is, from this point of view, not unnatural.

Perron [1] has proved theorems which may be derived from Theorems 1 and 2 if one regards zero as a quadratic residue. Thus, for $p=4 k-1$, he has shown that if $A$ is the set of quadratic residues, $\bmod p$, including zero, and if $a$ is any residue prime to $p$, the set $a+A$ consists of $k$ residues and $k$ nonresidues. This is also true of the set $A^{\prime}$ consisting of 0 and the quadratic nonresidues $\bmod p$. It would be interesting to know whether or not the sets $A$ and $A^{\prime}$ are the only sets with $2 k$ elements which have this property. Without substantial modification our method will not yield an answer to this question. An example given by Perron for the case $m=15$ shows, however, that the complete analogue of Theorem 4 is false; that is, the existence of sets with this property for an arbitrary modulus does not force this modulus to be a prime.

\section{REFERENCE}

1. O. Perron, Bemerkungen ïber die Verteilung der quadratische Reste, Math. Zeit. vol. 56 (1952) pp. 122-130.

Michigan State College 